\title{
Cyclosporin A preferentially attenuates skeletal slow-twitch muscle regeneration
}

E.H. Miyabara, M.S. Aoki and A.S. Moriscot
Departamento de Biologia Celular e do Desenvolvimento, Instituto de Ciências Biomédicas, Universidade de São Paulo, São Paulo, SP, Brasil

\author{
Correspondence \\ A.S. Moriscot \\ Departamento de Biologia Celular e \\ do Desenvolvimento, ICB I, USP \\ Av. Lineu Prestes, 1524 \\ 05508-900 São Paulo, SP \\ Brasil \\ Fax: +55-11-3091-7311 \\ E-mail: moriscot@usp.br \\ Presented at the XIX Annual \\ Meeting of the Federação de \\ Sociedades de Biologia Experimental, \\ Águas de Lindóia, SP, Brazil, \\ August 25-29, 2004. \\ Research supported by FAPESP. \\ E.H. Miyabara is the recipient of a \\ FAPESP fellowship \\ (No. 02/03195-2). \\ ........
}

Received April 14, 2004 Accepted January 18, 2005

\begin{abstract}
Calcineurin, a $\mathrm{Ca}^{2+} /$ calmodulin-dependent phosphatase, is associated with muscle regeneration via NFATc1/GATA2-dependent pathways. However, it is not clear whether calcineurin preferentially affects the regeneration of slow- or fast-twitch muscles. We investigated the effect of a calcineurin inhibitor, cyclosporin A (CsA), on the morphology and fiber diameter of regenerating slow- and fast-twitch muscles. Adult Wistar rats $(259.5 \pm 9 \mathrm{~g})$ maintained under standard conditions were treated with CsA ( $20 \mathrm{mg} / \mathrm{kg}$ body weight, $i p)$ for 5 days, submitted to cryolesion of soleus and tibialis anterior (TA) muscles on the 6th day, and then treated with CsA for an additional 21 days. The muscles were removed, weighed, frozen, and stored in liquid nitrogen. Cryolesion did not alter the body weight gain of the animals after 21 days of regeneration $(P=0.001)$ and $C s A$ significantly reduced the body weight gain $(15.5 \% ; \mathrm{P}=0.01)$ during the same period. All treated TA and soleus muscles showed decreased weights (17 and 29\%, respectively, $\mathrm{P}<0.05)$. CsA treatment decreased the cross-sectional area of both soleus and TA muscles of cryoinjured animals (TA: 2108 \pm 930 vs $792 \pm 640 \mu \mathrm{m}^{2}$; soleus: $2209 \pm 322$ vs $764 \pm 439 \mathrm{~m}^{2} ; \mathrm{P}<$ $0.001)$. Histological sections of both muscles stained with Toluidine blue revealed similar regenerative responses after cryolesion. In addition, CsA was able to minimize these responses, i.e., centralized nuclei and split fibers, more efficiently so in TA muscle. These results indicate that calcineurin preferentially plays a role in regeneration of slow-twitch muscle.
\end{abstract}

Skeletal muscle injury and subsequent regeneration are initially characterized by neutrophil infiltration and inflammation, followed by phagocytosis of necrotic debris by macrophages which have invaded the damaged region (1). Satellite cells, which are located between the basal lamina and sarcolemma of mature myofibers in a quiescent state (2), become activated in response to
Key words

- Calcineurin

- Cyclosporin A

- Regeneration

- Fast and slow twitch muscles growth factors and cytokines and undergo proliferation, migration and differentiation into myoblast-like cells (3). Subsequently, myoblasts fuse with each other to form young multinucleated myotubes, which frequently fuse with existing damaged myofibers (4).

Although much progress has been made in the understanding of skeletal muscle regeneration, knowledge of the signaling path- 
ways triggered by injury remains elusive. Calcineurin, a cytoplasmic calcium/calmodulin-dependent-phosphatase implicated in the signaling of fiber type (fast to slow) conversion (5) and heart hypertrophy (6), has been recently associated with muscle regeneration via the nuclear factor $\mathrm{c} 1$ of activated $\mathrm{T}$ cells (NFATc1)- and GATA2 (a zinc finger transcription factor)-dependent pathways (7). Furthermore, a previous study has demonstrated that treatment with cyclosporin A (CsA), an inhibitor of calcineurin, inhibits the regeneration of slow-twitch skeletal muscle, suggesting that calcineurin is important for skeletal muscle adaptation to injury in this muscle type (8).

It is well known that important differences exist between slow- and fast-twitch muscles. These include different patterns of motoneuron activation that stimulate distinctive programs of gene expression and consequently generate different amplitudes of $\mathrm{Ca}^{2+}$ transients during contraction of muscle fibers (9). In addition, CsA differentially affects muscle growth and maintenance depending on the skeletal muscle phenotype (10). Since it is unclear whether calcineurin is differentially involved in regeneration of slow- and fast-twitch muscles, we investigated here the effects of the calcineurin inhibitor CsA on the morphology and fiber cross-sectional area of fast- and slow-twitch muscles after regeneration induced by cryolesion.

The experimental protocols used in this study are in accordance with the ethical principles in animal research followed by the Brazilian College of Animals Experimentation (COBEA) and were approved by the Ethics Committee for Animals Research (CEEA) of the Institute of Biomedical Sciences, University of São Paulo.

Adult male Wistar rats ( $\mathrm{N}=12,8$ weeks of age) weighing 230-300 $\mathrm{g}$ were divided at random into groups and kept in a room at constant temperature $\left(25^{\circ} \mathrm{C}\right)$ with alternating 12-h periods of light and darkness and fed food (Purina ${ }^{\circledR}$ chow) and water ad libitum. Surgical procedures were performed under ketamine and xylazine anesthesia (30 and $10 \mathrm{mg} / \mathrm{kg}$ ip, respectively). The animals were weighed immediately before and after the experimental procedures and were killed with an overdose of ketamine and xylazine.

In all animals used in the present study, one soleus and one tibialis anterior (TA) muscles were cryolesioned (left leg) and the contralateral soleus and TA (right leg) were used as control. The animals were divided at random into 2 groups and one of them $(\mathrm{N}=$ 6) was cryolesioned and analyzed 21 days after injury. The other group $(\mathrm{N}=6)$ received CsA treatment starting 5 days prior to cryolesion and extending up to the end of the experiment (21 days after cryolesion). CsA administration was initiated 5 days prior to cryolesion to achieve proper intracellular drug accumulation. In one group of animals, CsA (Sandimmun, Novartis, Basel, Switzerland) diluted 1:5 with physiologic saline was administered ip twice a day at the dose of 10 $\mathrm{mg} / \mathrm{kg}$ body weight.

The animals were anesthetized and soleus and TA muscles were surgically exposed. Cryolesion consisted of two freezethaw cycles of the muscle in situ. Freezing was performed by applying the flat end $(0.4$ $\mathrm{x} 0.4 \mathrm{~cm}$ and $0.4 \mathrm{x} 1 \mathrm{~cm}$ for the soleus and TA muscles, respectively) of a piece of steel precooled in liquid nitrogen to the surface of the muscles and maintaining it in this position for $10 \mathrm{~s}$ (twice). After the muscles had thawed, the wounds were closed with polyamide sutures (6-0), and the animals kept for several hours on a warm plate $\left(37^{\circ} \mathrm{C}\right)$ to prevent hypothermia.

Animals were sacrificed and the left and right soleus and TA muscles were removed, weighed and immediately frozen in molten isopentane and stored in liquid nitrogen. Frozen muscles were cut into $10-\mu \mathrm{m}$ cross-sections with a cryostat (Leica CM3050, Nussloch, Germany). Unfixed histological sections were stained with aqueous Tolui- 
dine-blue-borax solution (both $1 \%, \mathrm{w} / \mathrm{v}$ ) for the visualization of general morphology $(11,12)$.

Muscle fiber cross-sectional area was evaluated on a digitizing unit connected to a computer (Image Pro-plus, Media Cybernetic, Silver Spring, MD, USA). Toluidineblue-borax was used to determine the crosssectional areas of 3000 TA fibers and 1500 soleus muscle fibers. Three cross-sections of the TA and soleus muscles from different animals were analyzed per group. The fiber cross-sectional areas of muscles treated with CsA and cryolesioned were measured close to the site of injury, therefore avoiding fibers which were not affected by cryolesion.

One-way analysis of variance (ANOVA) followed by the Tukey multiple comparison test was used to compare more than two groups, with the level of significance set at $\mathrm{P}$ $<0.05$.

As expected, after 21 days of regeneration, cryoinjured animals presented an increase in body weight $(14 \%, \mathrm{P}=0.001$, paired Student $t$-test; Table 1) compared to their respective controls and CsA significantly reduced body weight gain $(7.5 \% \mathrm{vs}$ initial body weight, and $15.5 \%$ vs final body weight of the cryolesioned group, $\mathrm{P}<0.05$, paired Student $t$-test; Table 1) during the same period.

The TA and soleus muscle weights significantly decreased in animals submitted only to cryolesion, only to CsA treatment and to cryolesion combined with CsA treatment compared to control muscles (TA: 16.4, 16 , and $18 \%$, respectively; soleus: $35,19.6$, and $33 \%$, respectively; $\mathrm{P}<0.05$; Table 1 ).

The fiber cross-sectional areas of the TA and soleus muscles of cryoinjured animals were decreased (27.5 and 50\%, respectively, $\mathrm{P}<0.001$; Table 1). In contrast, CsA treatment alone did not induce changes in soleus cross-sectional area (Table 1) and promoted a modest, although statistically significant, increase in fiber cross-sectional area of TA muscles $(17.6 \%, \mathrm{P}<0.001$; Table 1$)$. In addition, CsA treatment combined with cryolesion to further decreased the crosssectional area of both muscles as compared to animals submitted only to cryolesion ( 60\%, P<0.001; Table 1).

The muscles from the control groups (intact or treated with CsA) showed normal morphology, i.e., the presence of fibers with peripheral nuclei and a polygonal shape and with no signs of lesion (Figure 1A-D). In normal intact muscle the nuclei were non-

\begin{tabular}{|c|c|c|c|c|c|c|}
\hline Group & $\begin{array}{l}\text { Initial BW } \\
\text { (g) }\end{array}$ & $\begin{array}{c}\text { Final BW } \\
\text { (g) }\end{array}$ & $\begin{array}{l}\text { TA } \\
\text { (mg) }\end{array}$ & $\begin{array}{l}\mathrm{SOL} \\
\text { (mg) }\end{array}$ & $\begin{array}{c}\text { TA } \\
\left(\mu \mathrm{m}^{2}\right)\end{array}$ & $\begin{array}{l}\mathrm{SOL} \\
\left(\mu \mathrm{m}^{2}\right)\end{array}$ \\
\hline $\begin{array}{l}\text { Control } \\
\text { Cryo }\end{array}$ & $254 \pm 9$ & $290 \pm 16^{b}$ & $\begin{array}{l}530 \pm 31 \\
443 \pm 48^{*}\end{array}$ & $\begin{aligned} 117 & \pm 10 \\
76 & \pm 13^{* *}\end{aligned}$ & $\begin{array}{l}2108 \pm 930 \\
1530 \pm 649 * *\end{array}$ & $\begin{array}{l}2209 \pm 322 \\
1120 \pm 706^{* *}\end{array}$ \\
\hline $\begin{array}{l}\text { CsA } \\
\text { Cryo + CsA }\end{array}$ & $265 \pm 9$ & $245 \pm 9^{a, c}$ & $\begin{aligned} 445.5 & \pm 45^{*} \\
435 & \pm 59^{*}\end{aligned}$ & $\begin{array}{l}94 \pm 1^{*} \\
78 \pm 10^{* *}\end{array}$ & $\begin{aligned} 2480 & \pm 947^{* *} \\
792 & \pm 640^{* *}\end{aligned}$ & $\begin{aligned} 2370 & \pm 876 \\
764 & \pm 439 * *\end{aligned}$ \\
\hline
\end{tabular}

Data are reported as means \pm SD; $N=5-8$ for body weight (BW) and muscle weights and $N=3000$ and 1500 cells for fiber cross-sectional area of tibialis anterior (TA) and soleus (SOL) muscles, respectively. Rats were treated with cyclosporin A (CsA, $20 \mathrm{mg} / \mathrm{kg}$ body weight; ip) for 5 days. On the 6th day, they were submitted to cryolesion of SOL and TA muscles, and then treated with CsA for an additional 21 days.

One-way ANOVA was applied to test differences among 4 groups (control; Cryo: cryolesioned; CsA: treated with CsA; Cryo + CsA: cryolesioned-CsA treated). Significantly different from control: ${ }^{*} P<0.05$ and ${ }^{*} \mathrm{P}<0.001$. The paired Student $t$-test was applied to test differences between 2 groups (initial BW vs final BW; final BW of control vs final BW of treated group). Significantly different from initial BW: ${ }^{\mathrm{P}}=0.01$; $\mathrm{bP}=0.001$. Significantly different from final BW of control: ${ }^{\mathrm{C} P}=0.0005$. 

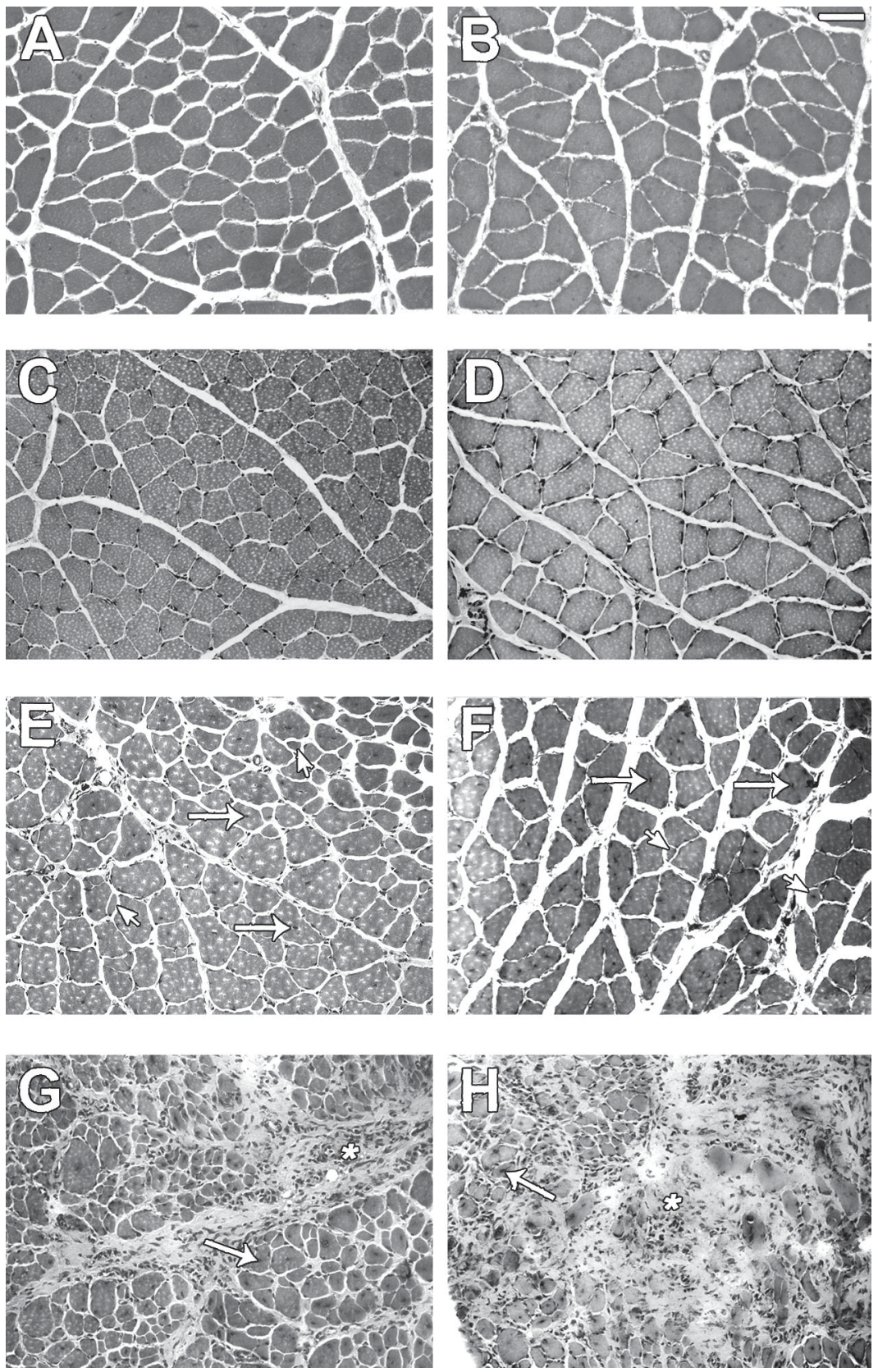

Figure 1. Histological features of tibialis anterior $(A, C, E$, and $G)$ and soleus $(B, D, F$, and $H)$ muscle cross-sections. $A$ and $B$, Control groups; $C$ and $D$, cyclosporin A-treated muscles; $E$ and $F$, cryolesioned groups regenerated after 21 days; $G$ and $H$, cyclosporin A-treated animals 21 days after cryolesion. Note that the control groups (A and B) and cyclosporin Atreated groups ( $C$ and $D$ ) have normal morphology. Regenerated tibialis anterior and soleus muscles ( $E$ and $F$, respectively) show fibers with centralized nuclei (pointed by arrows) and split fibers (pointed by arrowheads). In the group treated with cyclosporin A and analyzed at 21 days after cryolesion, both the tibialis anterior and soleus muscles showed deficient regeneration with the presence of extensive inflammatory cell infiltration (asterisk, $G$ and $\mathrm{H})$ and clear spaces among muscle fibers. Note the reduced presence of muscle fibers with centralized nuclei and a small cross-sectional area neighboring the central site of lesion in $\mathrm{G}$ and $\mathrm{H}$ (pointed by arrows) as compared to $\mathrm{E}$ and $\mathrm{F}$. Toluidine blue staining. Bar: $100 \mu \mathrm{m}$. peripheral in $\sim 1 \%$ of the fibers.

The soleus and TA muscles cryolesioned and analyzed at 21 days after injury showed regenerated muscle fibers with centrally located nuclei and split fibers (Figure 1E,F, respectively). Centrally nucleated muscle fibers and split fibers are hallmarks of muscle regeneration (13). In contrast, at the same time, the soleus and TA muscles of cryolesioned animals treated with CsA (Figure $1 \mathrm{G}, \mathrm{H})$ were greatly deficient in regenerated muscle fibers, as observed by the presence of extensive inflammatory cell infiltration and clear spaces among the muscle fibers at the site of lesion and by the reduced presence of muscle fibers with centralized nuclei and small cross-sectional areas. It is noteworthy that the TA muscles of cryolesioned animals treated with CsA showed fewer signs of injury (presence of inflammatory processes and clear spaces among muscle fibers) compared to soleus.

As previously demonstrated, the cryolesion model was effective in inducing injury and subsequent regeneration in a well-delimited area of the muscle belly (14) and did not alter body weight gain after 21 days (8). Furthermore, the dose of CsA $(20 \mathrm{mg} / \mathrm{kg}$ body weight ${ }^{-1}$ day $^{-1}$ ) used in the present study is known to cause a significant reduction in calcineurin activity ( 60\%) (15).

Cryolesion and CsA treatment, separately or combined, decreased the muscle weights at 21 days after cryolesion. In this period, affected muscles are still at $\sim 70 \%$ of their final weight, this being an appropriate time point to address regain of muscle mass in muscle regeneration.

Since CsA combined with cryolesion was able to further decrease cross-sectional area as compared to cryolesion alone, the present study strongly suggests that calcineurin is likely to be involved in repression of skeletal muscle fiber diameter regain during regeneration.

Currently it seems to be a consensus that calcineurin does not play an important role 
in skeletal muscle mass gain in overload models (16). Since our results clearly show that calcineurin is important to skeletal muscle weight regain after cryolesion, one might reason that skeletal muscle mass gain above the eutrophic status is probably controlled by different mechanisms as compared to the regain of muscle mass that occurs in immobilization/recovery models and after injury. Therefore, gain of muscle mass should not be necessarily considered a stereotypic response.

The better outcome of TA muscle than soleus indicates that the calcineurin pathway might be preferentially involved in regeneration of slow-twitch skeletal muscle. Indeed, a previous study has shown that calcineurin activity is significantly higher in slow-twitch muscles (10), suggesting that alternative intracellular pathways yet to be discovered might be important for skeletal muscle regeneration in a fiber type preferential manner.

In conclusion, our results indicate that calcineurin plays a preferential role in skeletal muscle regeneration of slow-twitch muscle.

\section{Acknowledgments}

We thank A.G. Soares Jr. and A.C. Partezani for excellent technical assistance.

\section{References}

1. Tidball JG (1995). Inflammatory cell response to acute muscle injury. Medicine and Science in Sports and Exercise, 27: 1022-1032.

2. Grounds MD \& Yablonka-Reuveni Z (1993). Molecular and cell biology of skeletal muscle regeneration. Molecular and Cell Biology of Human Diseases Series, 3: 210-256.

3. Allen RE \& Rankin LL (1990). Regulation of satellite cells during skeletal muscle growth and development. Proceedings of the Society for Experimental Biology and Medicine, 194: 81-86.

4. Seale P \& Rudnicki MA (2000). A new look at the origin, function, and "stem-cell" status of muscle satellite cells. Developmental Biology, 218: 115-124.

5. Chin ER, Olson EN, Richardson JA, Yang Q, Humphries C, Shelton J, Wu H, Zhu W, Bassel-Duby R \& Williams RS (1998). A calcineurindependent transcriptional pathway controls skeletal muscle fiber type. Genes and Development, 12: 2499-2509.

6. Molkentin JD, Lu JR, Antos CL, Markham B, Richardson J, Robbins J, Grant SR \& Olson EN (1998). A calcineurin-dependent transcriptional pathway for cardiac hypertrophy. Cell, 93: 215-228.

7. Sakuma K, Nishikawa J, Nakao R, Watanabe K, Totsuka T, Nakano $H$, Sano M \& Yasuhara M (2003). Calcineurin is a potent regulator for skeletal muscle regeneration by association with NFATc1 and GATA-2. Acta Neuropathologica, 105: 271-280.

8. Irintchev A, Zweyer M, Cooper RN, Butler-Browne GS \& Wernig A (2002). Contractile properties, structure and fiber phenotype of intact and regenerating slow-twitch muscles of mice treated with cyclosporin A. Cell and Tissue Research, 308: 143-156.

9. Olson EN \& Williams RS (2000). Remodeling muscles with calcineu- rin. Bioassays, 22: 510-519.

10. Mitchell PO, Mills ST \& Pavlath GK (2002). Calcineurin differentially regulates maintenance and growth of phenotypically distinct muscles. American Journal of Physiology, 282: C984-C992.

11. Morini CC, Pereira EC, Selistre de Araujo HS, Ownby CL \& Salvini TF (1998). Injury and recovery of fast and slow skeletal muscle fibers affected by ACL myotoxin isolated from Agkistrodon contortrix laticinctus (broad-banded copperhead) venom. Toxicon, 36: 10071024.

12. Salvini TF, Morini CC, Selistre de Araujo HS \& Ownby CL (1999). Long-term regeneration of fast and slow murine skeletal muscles after induced injury by ACL myotoxin isolated from Agkistrodon contortrix laticinctus (broad-banded copperhead) venom. Anatomical Record, 254: 521-533.

13. Karpati G, Carpenter S \& Prescott $S$ (1988). Small-caliber skeletal muscle fibers do not suffer necrosis in $\mathrm{mdx}$ mouse dystrophy. Muscle and Nerve, 11: 795-803.

14. Wernig A, Irintchev A \& Lange G (1995). Functional effects of myoblast implantation into histoincompatible mice with or without immunosuppression. Journal of Physiology, 484: 493-504.

15. Dunn SE, Simard AR, Bassel-Duby R, Williams RS \& Michel RN (2001). Nerve activity-dependent modulation of calcineurin signaling in adult fast and slow skeletal muscle fibers. Journal of Biological Chemistry, 276: 45243-45254.

16. Bodine SC, Latres E, Baumhueter $S$ et al. (2001). Akt/mTOR pathway is a crucial regulator of skeletal muscle hypertrophy and can prevent muscle atrophy in vivo. Nature Cell Biology, 3: 1014-1019. 